\title{
Manejo da sífilis na gestação: conhecimentos, práticas e atitudes dos profissionais pré-natalistas da rede SUS do município do Rio de Janeiro
}

\author{
Treatment of syphilis during pregnancy: knowledge, practices \\ and attitudes of health care professionals involved in antenatal care \\ of the Unified Health System (SUS) in Rio de Janeiro City
}

Rosa Maria Soares Madeira Domingues ${ }^{1}$

Lilian de Mello Lauria ${ }^{2}$

Valeria Saraceni ${ }^{2}$

Maria do Carmo Leal ${ }^{3}$

${ }^{1}$ Instituto de Pesquisa

Clínica Evandro Chagas,

Fiocruz. Avenida Brasil

4365, Manguinhos.

21040-360 Rio de Janeiro

RJ.rosa.domingues@

ipec.fiocruz.br

${ }^{2}$ Secretaria Municipal de

Saúde, Prefeitura da Cidade do Rio de Janeiro.

${ }^{3}$ Escola Nacional de Saúde

Pública Sérgio Arouca,

Fiocruz.

\begin{abstract}
This article seeks to evaluate knowledge, practices and attitudes of health care workers $(H C W)$ involved in antenatal care in the Unified Health System (SUS) in Rio de Janeiro City (RJC) and to identify major barriers to the implementation of treatment for syphilis in pregnancy care protocols. A cross-sectional study was conducted with $102 \mathrm{HCW}$ in antenatal care at SUS, corresponding to $70 \%$ of the eligible pool. Univariate and bivariate analysis were performed using SPSS version 16.0. A number of barriers were identified with respect to knowledge of and familiarity with the current protocols, difficulties related to DST management, relationship with patients and clinics organizational context, which were distinct according to the type of health unit. HCW who had greater access to training and technical manuals had a better performance, although the overall effect was discrete. Identifying barriers to adherence to health care protocols is essential to formulate intervention strategies. Access to protocols through training and technical manuals showed a discrete effect in the improvement of the care delivered to patients, pointing to the need of innovative ongoing education of $\mathrm{HCW}$.
\end{abstract}

Key words Syphilis, Antenatal care, Clinical protocols, Ongoing education
Resumo Este artigo objetiva avaliar os conhecimentos, as práticas e as atitudes dos profissionais pré-natalistas da rede de serviços públicos de saúde (SUS) do município do Rio de Janeiro (MRJ) e identificar as principais barreiras para a implantação dos protocolos assistenciais de manejo da sífilis na gestação. Estudo transversal com 102 profissionais pré-natalistas da rede SUS do MRJ, correspondendo a uma taxa de resposta de $70 \%$ dentre os elegíveis. Foi realizada análise uni e bivariada com utilização do software SPSS 16.0. Foram verificadas diversas barreiras relacionadas ao conhecimento e à familiaridade com os protocolos assistenciais, dificuldades na abordagem das DST, questões dos usuários e contexto organizacional, que apresentaram distribuição distinta segundo tipo de serviço de saúde. Profissionais com mais acesso a treinamentos e manuais técnicos apresentaram melhor desempenho, sendo esses efeitos discretos. A identificação de barreiras para a adoção de protocolos assistenciais é fundamental para a formulação de estratégias de intervenção. O acesso ao conteúdo dos protocolos por treinamentos $e$ manuais técnicos mostraram efeito discreto na melhoria das condutas assistenciais, sendo necessárias outras abordagens de educação continuada dos profissionais.

Palavras-chave Sífilis, Assistência pré-natal, Protocolos clínicos, Educação continuada 


\section{Introdução}

A sífilis congênita (SC) permanece como um grave problema de saúde pública no Brasil ${ }^{1} \mathrm{e}$ em outros países da América Latina ${ }^{2}$. Dados nacionais revelam a ocorrência de 54.141 casos de sífilis congênita no período de 2000 a junho de 2010, com uma incidência, em 2009, de 1,7 casos por 1.000 nascidos vivos no país ${ }^{1}$. Considerando os resultados do último estudo sentinela realizado em 2004, que encontrou uma prevalência de sífilis na gestação de $1,6 \%$, estima-se uma subnotificação de $67 \%$ dos casos de SC a cada ano, indicando uma situação ainda mais grave ${ }^{3}$.

O estado do Rio de Janeiro apresentou o maior número de casos acumulados no período, 11.741 , correspondendo a $21,7 \%$ dos casos detectados no país ${ }^{1}$. No município do Rio de Janeiro (MRJ), a incidência manteve-se estável nos últimos dez anos, com taxas próximas a 10 por 1.000 nascidos vivos ${ }^{4}$, valor dez vezes superior à meta de eliminação proposta pelo Ministério da Saúde em $1997^{5}$. Além do elevado número de casos, observa-se a ocorrência de formas graves da doença, sendo a SC um importante componente da mortalidade perinatal nesse município ${ }^{6}$.

Nos demais países da América Latina e Caribe, dados de notificação revelam uma prevalência elevada da sífilis na gestação para o ano 2002, com uma taxa global de $3,1 \%$, variando de $1 \%$ e $6 \%$ entre os países da região, sendo a incidência da SC também elevada, de 1,4 a 12 por mil nascidos vivos ${ }^{2}$.

A SC é uma doença que pode ser evitada com recursos disponíveis na assistência pré-natal ${ }^{7,8}$. Entretanto, estudos nacionais e internacionais apontam para falhas nesta, com oportunidades perdidas no diagnóstico e tratamento dos ca$\operatorname{sos}^{9,10}$. Do total de casos notificados no país, em $2009,75,5 \%$ das gestantes haviam recebido assistência pré-natal, mas apenas $55,4 \%$ tiveram diagnóstico de sífilis na gestação e a maioria dos parceiros não recebeu tratamento ${ }^{1}$. No município do Rio de Janeiro, dados provenientes dos relatórios dos grupos de investigação de SC no período 1999-2004, também revelam falhas na assistência, com realização do exame sorológico de triagem por apenas $75 \%$ das gestantes, tratamento de $85 \%$ dos casos de sífilis na gestação e de apenas $25 \%$ dos parceiros ${ }^{11}$.

Estudo da trajetória assistencial de gestantes com assistência pré-natal na rede SUS do MRJ que apresentaram um caso de SC como desfecho da gestação ${ }^{12}$ encontrou diversos problemas relacionados ao diagnóstico da infecção durante a gestação, com 25\% das gestantes apresentando diagnóstico da sífilis apenas na internação para o parto; falhas no tratamento da gestante; e ausência de tratamento do(s) parceiro(s) em 100\% dos casos. Foi observada taxa de transmissão vertical de 38,5\%, incidência de SC de 6 casos por 1000 gestantes, formas graves da doença e proporção elevada de prematuridade e de baixo peso ao nascer. Gestantes com diagnóstico de sífilis apresentavam pior condição socioeconômica e de acesso aos serviços de saúde, dado também observado em estudos anteriores ${ }^{6,13}$.

Resultados de revisões sistemáticas recentes reforçam a validade dos protocolos assistenciais vigentes e a importância do tratamento das gestantes com diagnóstico de sífilis na gravidez, principalmente na fase ativa da doença, para a redução de casos de $\mathrm{SC}^{14}$ e de desfechos perinatais negativos tais como natimortalidade ${ }^{15}$, prematuridade e mortalidade neonatal ${ }^{14}$.

Os protocolos assistenciais têm sido uma ferramenta utilizada com frequência para a melhoria da qualidade dos serviços e maior efetividade da assistência. Entretanto, seu impacto é variado e estudos de avaliação da implantação de protocolos assistenciais indicam que diversos fatores podem dificultar ou impedir sua adoção. Estes podem estar relacionados a características do protocolo em si, à sua forma de implantação, ao profissional de saúde, ao paciente ou ao ambiente externo ${ }^{16,17}$, bem como à existência de incentivos e mecanismos regulatórios ${ }^{18}$. Além disso, as barreiras à implantação se modificam em função do protocolo clínico e mesmo entre diretrizes assistenciais de um mesmo protocolo, sendo fundamental a identificação dessas barreiras para a adoção de estratégias específicas ${ }^{19-21}$.

Considerando as oportunidades perdidas no diagnóstico e tratamento das gestantes durante a assistência pré-natal, é fundamental identificar as principais barreiras que têm impedido a adoção dos protocolos assistenciais disponíveis durante a assistência pré-natal. Este estudo tem por objetivo verificar os conhecimentos, as práticas e as atitudes (CPA) dos profissionais de saúde que atuam na assistência pré-natal da rede de serviços do Sistema Único de Saúde (SUS) do MRJ em relação ao manejo da sífilis na gestação, as principais barreiras percebidas por esses profissionais para a implantação dos protocolos assistenciais e suas sugestões para a melhoria da assistência. 


\section{Métodos}

Trata-se de um inquérito tipo CPA com profissionais de saúde de nível superior que atuam na assistência pré-natal de unidades do SUS do MRJ. Este estudo é parte de um projeto maior, de avaliação da qualidade da assistência pré-natal na rede SUS do Município do Rio de Janeiro, coordenado pelo Departamento de Epidemiologia e Métodos Quantitativos em Saúde da Escola Nacional de Saúde Pública Sérgio Arouca da Fiocruz ${ }^{12}$.

O município do Rio de Janeiro é a segunda maior cidade brasileira, com cerca de 6 milhões de habitantes. Possui uma extensa rede de serviços públicos de saúde que presta assistência a cerca de $70 \%$ da população do município. O prénatal é realizado em unidades hospitalares, unidades básicas de saúde (UBS), unidades de saúde da família (USF) e em uma Casa de Parto (CP).

Para o estudo principal, foi realizada uma amostragem por conglomerado em dois estágios, sendo selecionados, no primeiro, os estabelecimentos de saúde da rede SUS do MRJ e, no segundo, as gestantes. As unidades primárias de seleção foram estratificadas, segundo tipo de unidade em: Hospitais/maternidades, UBS, USF e $\mathrm{CP}^{12}$.

Para este subprojeto, foram considerados elegíveis todos os médicos e enfermeiros que realizavam consulta individual de pré-natal nas unidades selecionadas para o estudo principal, sendo identificados 144 profissionais de saúde.

Utilizou-se um questionário estruturado, autoaplicado, elaborado especificamente para este estudo. Para orientar a construção do instrumento, foram realizados dois grupos focais, um com profissionais de saúde e outro com gestores, em julho de 2007. O questionário continha questões relacionadas à: a) formação dos profissionais, tempo de atuação e acesso a treinamentos e manuais normativos; b) informações sobre características das unidades em relação à disponibilidade de exames para diagnóstico da sífilis e do HIV, medicamentos para tratamentos da sífilis, protocolos assistenciais e ações de vigilância epidemiológica; e c) percepções do profissional em relação à infecção pela sífilis e pelo HIV durante a gestação e suas sugestões para melhorias da assistência visando melhor controle dessas afecções.

Os questionários foram entregues aos profissionais de saúde pelos supervisores de campo, sendo garantido o anonimato. Foi estabelecido um prazo inicial de uma (01) semana para o preenchimento do questionário pelos profissionais selecionados, prorrogado por mais uma. Questionários não preenchidos ao final de quinze dias foram considerados como recusa. Foi preenchida uma planilha de campo, que permitiu calcular a taxa de resposta por serviço de saúde.

Todos os questionários foram revisados por um dos pesquisadores. Os dados dos questionários foram digitados em banco de dados próprio do estudo, por um único digitador.

$\mathrm{Na}$ fase de análise, foi feita inicialmente uma descritiva das características dos profissionais de saúde, em relação a sua formação profissional, especialização, local e tempo de atuação. Os estudos de CAP objetivam reconhecer o que as pessoas sabem sobre determinado tema; o que sentem e pensam sobre esse tema; e a maneira como demonstram seus conhecimentos e atitudes por meio de suas ações. Para avaliação do conhecimento dos protocolos assistenciais, verificou-se o acesso a treinamentos e materiais normativos do Ministério da Saúde e para avaliar a familiaridade com o conteúdo desses protocolos verificou-se a proporção de acerto em relação a um caso clínico apresentado. Avaliou-se também o conhecimento e a concordância dos profissionais em relação a duas inovações assistenciais adotadas pela SMS/RJ visando o melhor controle da sífilis na gestação: relatório de investigação de casos de SC (baseado na proposta do Ministério da Saúde de grupos de investigação de SC, que tem por objetivo o melhor monitoramento da testagem sorológica para sífilis e do tratamento das gestantes infectadas e seus parceiros); e implantação de testes rápidos treponêmicos para confirmação do diagnóstico da sífilis em gestantes com exames VDRL com titulação menor ou igual a 1/ 8. A adequação da prática dos profissionais na assistência pré-natal foi aferida pelo cumprimento das rotinas assistenciais preconizadas: solicitação dos exames sorológicos de rotina (VDRL) no $1^{\circ}$ e $3^{\circ}$ trimestre gestacional, prescrição de tratamento para as gestantes com diagnóstico de sífilis, abordagem dos parceiros, e notificação de casos à Secretaria Municipal de Saúde. Para avaliação da atitude dos profissionais em relação à infecção pela sífilis na gestação e aos protocolos recomendados, verificou-se sua percepção em relação à vulnerabilidade das mulheres às Doenças Sexualmente Transmissíveis (DST); situação epidemiológica da Sífilis Congênita na cidade, visando verificar sua percepção em relação à gravidade da doença na cidade; concordância em relação aos protocolos assistenciais do Ministério da Saúde; dificuldades na abordagem das DST e sugestões de estratégias para melhoria da assistência.

Para identificação das barreiras ao cumprimento dos protocolos assistenciais, utilizou-se o 
modelo proposto por Cabana et al. ${ }^{19}$, que define os principais tipos de barreira como: a) barreiras relacionadas ao conhecimento: falta de conhecimento da existência do protocolo e falta de familiaridade com o seu conteúdo; b) barreiras relacionadas às atitudes: não concordância com o protocolo, não acreditar nos resultados a serem obtidos com o protocolo, inércia para modificar práticas pré-existentes, não se sentir capaz de atuar como o protocolo exige (questões ligadas à abordagem de temas ligados à sexualidade e às doenças sexualmente transmissíveis, por exemplo); c) barreiras relacionadas à prática: fatores externos, que podem estar relacionados ao paciente (não adesão, comorbidades), ao próprio protocolo (complexidade, dificuldade de utilização) ou ao ambiente organizacional (falta de insumos, tempo, apoio, etc...). Utilizou-se o teste qui-quadrado para verificar se as barreiras identificadas apresentaram diferença segundo o tipo de serviço de saúde onde o profissional prestava assistência prénatal (hospitais, UBS, USF/Casa de Parto).

Por último, verificou-se se características dos profissionais, relacionadas à sua formação, tempo de atuação e acesso a manuais e treinamentos, estavam associados ao maior conhecimento e familiaridade com o protocolo e maior adesão às práticas recomendadas, sendo realizada análise bivariada com aplicação do teste estatístico qui-quadrado para verificar diferenças entre proporções.

Para a realização da análise estatística no estudo principal, cada elemento da amostra de gestante recebeu uma ponderação pelo inverso da sua probabilidade de seleção e uma calibração para restituir a distribuição conhecida das consultas de pré-natal (dados da SMS/RJ para o ano 2006). Esse mesmo procedimento foi adotado em relação à amostra de profissionais de saúde, pois se espera que o número de consultas seja proporcional à disponibilidade de profissionais pré-natalistas em cada unidade. Para a amostra de profissionais, não foi possível a inclusão do efeito de desenho na análise, pois o questionário não continha o nome da unidade de saúde para garantir o sigilo do profissional, não sendo possível identificar o conglomerado. Entretanto, espera-se um efeito de desenho pequeno, já que o número de profissionais entrevistados em cada unidade foi inferior a dez. A análise dos dados foi realizada por meio dos softwares SPSS 16.0.

Este projeto foi aprovado pelo Comitê de Ética em Pesquisa da Secretaria Municipal de Saúde do Rio de Janeiro. Para garantir o anonimato dos profissionais, não foi utilizado o Termo de Consentimento Livre e Esclarecido assinado, para evi- tar sua identificação. Em seu lugar, utilizou-se uma carta-convite, informando os objetivos do estudo, a identificação dos pesquisadores e as instituições envolvidas, o caráter voluntário da pesquisa e a garantia do anonimato. Como o questionário dos profissionais era autoaplicado, considerou-se que o preenchimento e a devolução do mesmo expressava o consentimento do profissional em participar da pesquisa. Todos os cuidados foram adotados visando garantir o sigilo e a confidencialidade das informações obtidas.

\section{Resultados}

Dos 144 profissionais elegíveis, 139 (96,5\%) foram convidados a participar do estudo e receberam o questionário. Cinco profissionais (3,5\%) não foram abordados pela equipe da pesquisa por se encontrarem de férias ou licença. Dos que receberam o questionário, 102 o devolveram preenchido, correspondendo a 70\% dos elegíveis (Figura 1). Do grupo analisado, aproximadamente $70 \%$ eram médicos e $30 \%$ enfermeiros e $72,6 \%$ apresentavam especialização em saúde da

Profissionais elegíveis (144)

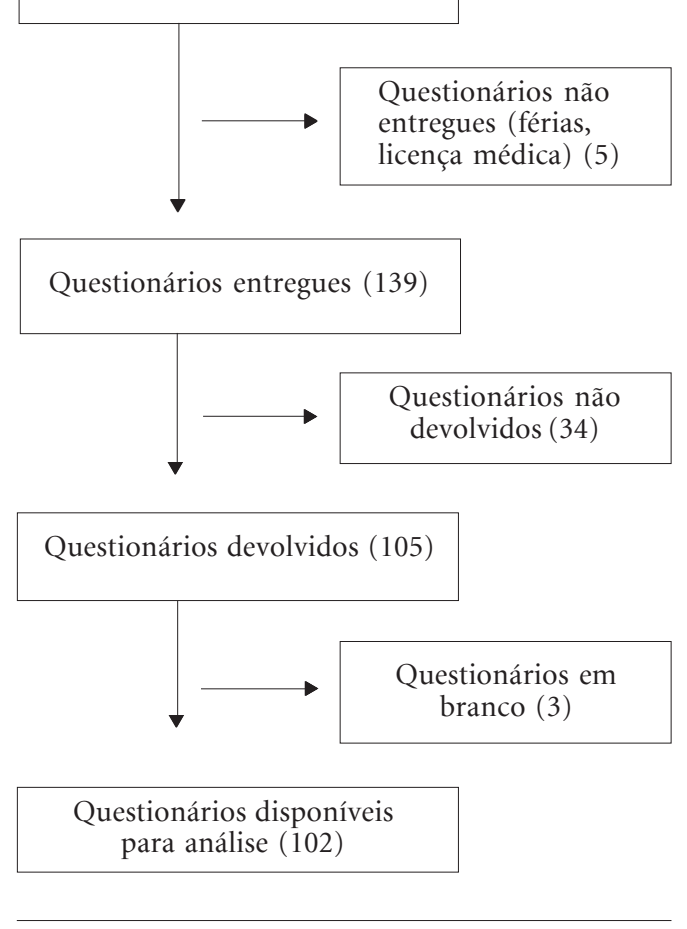

Figura 1. Fluxograma de retorno dos questionários. 


\section{Conhecimentos}

Uma elevada proporção de profissionais, superior a $70 \%$, relatava já ter participado de treinamentos sobre manejo da sífilis na gravidez, a maioria nos últimos cinco anos (dado não mostrado em tabela). Menor proporção foi observada em profissionais que atuavam em ESF/Casa de Parto, alcançando apenas 50\%. O acesso aos manuais técnicos do Ministério da Saúde também foi elevado, próximo a $80 \%$, sendo a leitura completa desses materiais referida por apenas $57,7 \%$ dos profissionais (Tabela 2).

Para avaliar a familiaridade com o protocolo, em particular com as condutas preconizadas para o tratamento das gestantes com diagnóstico de sífilis, foi apresentado um caso clínico de uma gestante com VDRL reagente, com título baixo, sem antecedentes de tratamento para sífilis. Considerou-se como resposta correta a pres-

Tabela 1. Características dos 102 profissionais prénatalistas. Município do Rio de Janeiro, 2007 2008 .

\begin{tabular}{lcc}
\hline \multicolumn{1}{c}{ Característica do profissional } & n & $\%$ \\
\hline Formação & & \\
$\quad$ Médico(a) & 71 & 69,6 \\
$\quad$ Enfermeiro(a) & 31 & 30,4 \\
Especialização em obstetrícia/saúde & & \\
da mulher & & \\
$\quad$ Sim & 74 & 72,6 \\
$\quad$ Não & 16 & 27,4 \\
Tempo de formado & & \\
$\quad<10$ anos & 23 & 22,5 \\
$\quad 10$ anos ou mais & 79 & 77,5 \\
Tempo de atuação como pré-natalista & & \\
$\quad<10$ anos & 48 & 47,1 \\
$\quad 10$ anos ou mais & 52 & 50,8 \\
Tempo de atuação na SMS/RJ & & \\
$\quad<10$ anos & 45 & 43,8 \\
$\quad 10$ anos ou mais & 56 & 54,7 \\
Unidade de atuação & & \\
$\quad$ Hospital & 30 & 30,1 \\
$\quad$ Unidade Básica de Saúde & 55 & 54,1 \\
$\quad$ Unidade de Saúde da Família/Casa & 16 & 15,8 \\
de parto & & \\
\hline & &
\end{tabular}

crição de tratamento para sífilis de duração ignorada, com penicilina $G$ benzatina dose total 7.200.000 U, sem necessidade de confirmação diagnóstica com teste treponêmico, conforme protocolo do Ministério da Saúde ${ }^{8}$. Observou-se uma proporção de acerto de apenas 50\%, sendo a maior parte do erro decorrente de solicitação de uma nova avaliação diagnóstica.

Quando questionados sobre as inovações adotadas pela SMS/RJ, 43,0\% dos profissionais referiam conhecer e aprovar os relatórios de investigação de sífilis congênita, mas 57,0\% referiam não conhecer essa estratégia. Aproximadamente $68,7 \%$ conheciam e aprovavam os testes rápidos para sífilis e $28,3 \%$ desconheciam sua implantação (Tabela 2).

\section{Atitudes}

Não foram relatadas discordâncias em relação aos protocolos assistenciais do Ministério da Saúde (Tabela 2).

97,9\% dos profissionais apresentavam conhecimento correto das formas de transmissão da sífilis, ou seja, vulnerabilidade aumentada de todas as pessoas com vida sexual ativa e relações sexuais desprotegidas, sem preconceitos em relação a número de parceiros ou situação socioeconômica (dado não mostrado em tabela).

Em relação à sífilis congênita, apenas 53,5\% apresentavam conhecimento correto sobre a situação epidemiológica dessa doença (Tabela 2) e $31,2 \%$ não sabiam qual era a situação da SC no RJ no momento da pesquisa (dado não mostrado em tabela).

Algum tipo de dificuldade para abordagem das DST foi relatada por $47 \%$ dos profissionais quando questionados sobre sua prática no manejo clínico da sífilis. Aproximadamente 40\% referiam dificuldade para conversar sobre sífilis com o parceiro e $15,4 \%$ para orientar sobre questões relacionadas às DST (forma de contágio, consequências para a saúde, tratamento, uso do preservativo).

\section{Práticas}

A solicitação dos dois exames de triagem de rotina foi relatada por $95 \%$ dos profissionais, sendo verificado erro na época de solicitação do segundo exame, que deveria ser feita no início do terceiro trimestre gestacional, em 35\% dos profissionais (dado não mostrado em tabela).

O tratamento das gestantes com sífilis é realizado na própria unidade de saúde, não haven- 
Tabela 2. Barreiras para o manejo adequado da sífilis na gestação segundo tipo de unidade de saúde. Município do Rio de Janeiro, 2007-2008.

\begin{tabular}{|c|c|c|c|c|c|c|c|c|c|}
\hline \multirow[t]{2}{*}{ Barreiras/Tipo de Unidade } & \multicolumn{2}{|c|}{$\begin{array}{c}\text { Total } \\
(\mathbf{n}=101)^{\mathrm{a}}\end{array}$} & \multicolumn{2}{|c|}{$\begin{array}{c}\text { Hospital } \\
(\mathbf{n}=30)\end{array}$} & \multicolumn{2}{|c|}{$\begin{array}{c}\text { UBS }^{b} \\
(n=55)\end{array}$} & \multicolumn{2}{|c|}{$\begin{array}{c}\mathrm{USF}^{\prime} \mathrm{CP}^{\mathrm{c}} \\
(\mathrm{n}=16)\end{array}$} & \multirow[t]{2}{*}{ p valor ${ }^{d}$} \\
\hline & $\mathbf{n}$ & $\%$ & $\mathbf{n}$ & $\%$ & $\mathbf{n}$ & $\%$ & $\mathbf{n}$ & $\%$ & \\
\hline \multicolumn{10}{|l|}{ Conhecimento } \\
\hline \multicolumn{10}{|l|}{ Conhecimento protocolo MS e } \\
\hline Participação em treinamentos & 78 & 77,2 & 26 & 86,7 & 44 & 80,0 & 8 & 50,0 & 0,014 \\
\hline Acesso a manuais técnicos & 79 & 78,2 & 22 & 73,3 & 44 & 80,0 & 13 & 81,2 & 0,738 \\
\hline Leitura completa dos Manuais ${ }^{\mathrm{f}}$ & 45 & 57,7 & 10 & 45,5 & 29 & 65,9 & 6 & 50,0 & 0,240 \\
\hline \multicolumn{10}{|l|}{ Conhecimento protocolos SMS/RJ ${ }^{g}$} \\
\hline Relatórios Sífilis Congênita & 43 & 43,0 & 10 & 33,3 & 27 & 50,0 & 6 & 37,5 & 0,298 \\
\hline Teste rápido para sífilis & 68 & 68,7 & 22 & 73,3 & 38 & 71,7 & 8 & 50,0 & 0,143 \\
\hline Acerto caso clínico SC ${ }^{\mathrm{h}}$ & 47 & 48,5 & 10 & 33,3 & 33 & 61,1 & 4 & 30,8 & 0,020 \\
\hline \multicolumn{10}{|l|}{ Atitude } \\
\hline Conhecimento situação SC & 54 & 53,5 & 20 & 66,7 & 26 & 47,3 & 8 & 50,0 & 0,220 \\
\hline Discordância protocolo MS & 0 & - & 0 & - & 0 & - & 0 & - & - \\
\hline Dificuldades abordagem DST ${ }^{\mathrm{i}}$ & 47 & 47,0 & 10 & 33,3 & 27 & 50,0 & 10 & 62,5 & 0,136 \\
\hline \multicolumn{10}{|l|}{ Prática } \\
\hline \multicolumn{10}{|l|}{ Fatores dos usuários } \\
\hline Início tardio pré-natal & 76 & 75,2 & 24 & 80,0 & 44 & 80,0 & 8 & 50,0 & 0,039 \\
\hline Não comparecimento dos parceiros & 53 & 53,0 & 16 & 53,3 & 30 & 55,6 & 7 & 43,8 & 0,707 \\
\hline Não adesão das gestantes & 34 & 34,3 & 6 & 20,0 & 24 & 44,4 & 4 & 26,7 & 0,062 \\
\hline \multicolumn{10}{|l|}{ Contexto organizacional } \\
\hline Testagem para sífilis & 50 & 50,0 & 6 & 20,0 & 39 & 72,2 & 5 & 31,2 & $<0,001$ \\
\hline Aplicação Penicilina G Benzatina & 0 & - & 0 & - & 0 & - & 0 & - & - \\
\hline Referência para parceiros & 18 & 17,8 & 4 & 13,3 & 14 & 25,5 & 0 & - & 0,013 \\
\hline
\end{tabular}

${ }^{a}$ Um dos profissionais não apresentava identificação do local de atuação. ${ }^{b}$ UBS $=$ Unidade Básica de Saúde; ${ }^{c}$ USF $=$ Unidade de Saúde da Família e CP = Casa de parto; ${ }^{\mathrm{d}}$ teste estatístico qui-quadrado; ${ }^{\mathrm{e}} \mathrm{MS}=$ Ministério da Saúde; ${ }^{\mathrm{f}}$ apenas para os profissionais que tiveram acesso aos manuais; ${ }^{\mathrm{S}} \mathrm{SMS} / \mathrm{RJ}=$ Secretaria Municipal de Saúde do Rio de Janeiro; ${ }^{\mathrm{h}} \mathrm{SC}=$ Sífilis Congênita; ${ }^{\mathrm{i}} \mathrm{DST}=$ Doença Sexualmente Transmissível

do relato de dificuldades na aplicação de penicilina benzatina (Tabela 2).

Em relação à abordagem dos parceiros das gestantes com DST, 35\% dos profissionais referiram que essa era realizada pelo próprio prénatalista. Metade dos profissionais apresentava uma conduta em relação aos parceiros que não seria a mais adequada, ou não abordando $(0,3 \%)$, ou enviando pedido de exames $(44,6 \%)$ ou tratamento $(3,5 \%)$ por meio da gestante (dado não mostrado em tabela).

Quase 90\% dos profissionais relataram que a unidade realizava notificação da sífilis na gestação, sendo em 59,8\% dos casos pelo próprio prénatalista.

Para os profissionais de saúde, as principais barreiras para a abordagem da sífilis relacionamse ao início tardio do pré-natal (75,2\%); não comparecimento dos parceiros ao serviço $(53,0 \%)$; dificuldades na testagem para sífilis $(34,7 \%)$, por demora no resultado do exame ou por falta de acesso ao teste treponêmico confirmatório; não adesão das gestantes à realização dos exames e/ ou do tratamento $(34,3 \%)$, e falta de referência para encaminhamento do parceiro $(17,3 \%)$.

Para melhor assistência aos casos de sífilis na gestação, as principais estratégias sugeridas foram a realização de trabalhos educativos com a população geral $(81,5 \%)$, facilitação do diagnóstico e tratamento dos parceiros $(80,4 \%)$, garantia de exames de qualidade $(60 \%)$, e treinamentos em aconselhamento $(62,7 \%)$ e manejo clínico $(65,3 \%)$. Para a maioria dos profissionais, treinamentos curtos, realizados no próprio serviço, seriam a modalidade preferencial de capacitação. Apenas 20\% dos profissionais consideraram o envio de informações sobre os casos ocorridos na cidade e no próprio serviço importante para o melhor controle desses agravos.

Na Tabela 2 são apresentadas de forma sumarizada as principais barreiras identificadas e sua variação conforme o tipo de unidade onde o profissional atuava. Notam-se diferenças, segundo o tipo de serviço de saúde, no acesso a treina- 
mentos, na familiaridade com o conteúdo dos protocolos (medido pelo acerto do caso clínico), no acesso precoce das gestantes ao pré-natal, na testagem para sífilis e na disponibilidade de referências para os parceiros.

A análise da adequação dos procedimentos em relação às características dos profissionais revelou que a formação médica e a especialização em obstetrícia/saúde da mulher estiveram associadas ao menor relato de dificuldades para a abordagem das Doenças Sexualmente Transmissíveis (DST). Verificou-se maior adequação na abordagem dos parceiros em profissionais sem especialização em obstetrícia/saúde da mulher e em profissionais que atuavam em USF/Casa de Parto. Profissionais com maior tempo de formado referiram maior adequação na solicitação dos exames de rotina e na prescrição de tratamento para a gestante, bem como menor dificuldade para a abordagem das DST. De forma semelhante, profissionais com maior tempo de atuação na SMS/RJ apresentaram maior proporção de conhecimento adequado sobre a situação da sífilis congênita e de prescrição de tratamento adequado para as gestantes, tendo também menor relato de dificuldade na abordagem das DST. Os treinamentos estiveram associados apenas a um conhecimento mais adequado sobre a situação da SC, com uma significância limítrofe. O acesso aos manuais técnicos mostrou-se associado ao tratamento adequado das gestantes, enquanto a leitura completa desses materiais esteve associada a maior adequação do conhecimento da situação epidemiológica, solicitação de exames e menor dificuldade de abordagem das DST. Profissionais que atuavam em Unidades Básicas de Saúde apresentaram também maior adequação na prescrição de tratamento para as gestantes (Tabela 3).

\section{Discussão}

No modelo desenvolvido por Cabana et al. ${ }^{19}$, utilizado neste estudo, a adesão dos profissionais aos protocolos assistenciais é considerada fundamental para transformar recomendações em resultados mais favoráveis. Segundo esse modelo, a falta de conhecimento e a falta de familiaridade afetam o conhecimento do profissional em relação a um protocolo assistencial. Para as atitudes, a falta de concordância com as recomendações, de autoconfiança para segui-las, a baixa expectativa em relação aos resultados que serão alcançados e a inércia para modificar prá- ticas anteriores são barreiras potenciais à implantação das recomendações. Independente do conhecimento e atitude do profissional, barreiras externas - relacionas ao usuário, ao contexto organizacional, ou às características do próprio protocolo - podem afetar sua habilidade em seguir as recomendações.

Neste estudo, foram identificadas falhas nas práticas adotadas durante a assistência pré-natal para o adequado manejo da sífilis na gestação, relacionadas principalmente à solicitação do segundo exame de rotina e problemas na abordagem dos parceiros. O baixo percentual de acerto do tratamento adequado das gestantes com exame de VDRL com titulação baixa também indica problemas na assistência das gestantes com esse quadro clínico.

Verificaram-se barreiras relacionadas tanto ao conhecimento dos protocolos, quanto às atitudes dos profissionais e à sua prática. A baixa familiaridade dos profissionais com o conteúdo do protocolo (conhecimento), dificuldades na abordagem das DST (atitude) e barreiras externas (prática), relacionadas aos usuários (início tardio do pré-natal, não adesão às recomendações, não comparecimento dos parceiros) e ao contexto organizacional (dificuldades para a testagem das gestantes e para tratamento dos parceiros) foram as mais relatadas.

A falta de concordância com os protocolos assistenciais não se mostrou uma barreira importante, mas o desconhecimento dos protocolos adotados nos últimos anos pela SMS/RJ constitui-se em um bom exemplo de como a falta de conhecimento impede a utilização plena das diretrizes assistenciais. A falta de acesso a exames treponêmicos, que são utilizados para confirmação da infecção pela sífilis, foi indicada como problema para o manejo da sífilis por mais de um terço dos profissionais entrevistados. Entretanto, a realização rotineira desses exames pelos laboratórios da SMS/RJ, quando o VDRL apresenta titulação inferior a 1/8, foi adotada em 2006, e cerca de um terço dos profissionais desconhecia essa rotina. Falhas na divulgação dessa inovação assistencial, elevada rotatividade dos profissionais e falta de confiança nessa metodologia diagnóstica, são fatores a serem investigados.

A análise das barreiras identificadas, segundo tipo de serviço, demonstra que algumas apresentam distribuição diferenciada e estão provavelmente relacionadas à infraestrutura das unidades ou ao seu modelo organizacional. Por exemplo, menor relato de barreiras em relação aos exames para sífilis em unidades hospitalares, 
Tabela 3. Adequação dos procedimentos de controle da infecção pela sífilis na gestação segundo características profissionais e de acesso a treinamentos e manuais técnicos. Município do Rio de Janeiro, 2007-2008.

\begin{tabular}{|c|c|c|c|c|c|c|c|c|c|}
\hline \multirow{2}{*}{ Exposição/desfecho } & \multicolumn{3}{|c|}{$\begin{array}{l}\text { Conhecimento } \\
\text { situação SC }\end{array}$} & \multicolumn{3}{|c|}{$\begin{array}{l}\text { Solicitação } \\
\text { VDRL }^{\text {b }}\end{array}$} & \multicolumn{3}{|c|}{$\begin{array}{l}\text { Tratamento } \\
\text { adequado }^{c}\end{array}$} \\
\hline & $\mathbf{n}$ & $\%$ & p valor & $\mathbf{n}$ & $\%$ & p valor & & $\%$ & $\mathrm{p}$ valor \\
\hline \multicolumn{10}{|l|}{ Formação } \\
\hline Médico $(\mathrm{n}=71)$ & 42 & 60,0 & & 45 & 63,4 & & & 3245,1 & \\
\hline Enfermeiro $(\mathrm{n}=31)$ & 13 & 41,9 & 0,093 & 21 & 67,7 & 0,672 & & $18 \quad 60,0$ & 0,170 \\
\hline \multicolumn{10}{|c|}{ Especialização obstetrícia/saúde da mulher } \\
\hline $\operatorname{Sim}(\mathrm{n}=74)$ & 44 & 58,7 & & 43 & 58,1 & & & $38 \quad 52,1$ & \\
\hline Não $(\mathrm{n}=16)$ & 6 & 37,5 & 0,122 & 12 & 70,6 & 0,343 & & 850,0 & 0,882 \\
\hline \multicolumn{10}{|l|}{ Tempo de formação } \\
\hline$<10$ anos $(\mathrm{n}=23)$ & 8 & 36,4 & & 10 & 43,5 & & & $\begin{array}{ll}5 & 22,7\end{array}$ & \\
\hline 10 anos ou $+(\mathrm{n}=79)$ & 47 & 58,8 & 0,062 & 56 & 70,0 & 0,019 & & 4456,4 & 0,005 \\
\hline \multicolumn{10}{|l|}{ Tempo de atuação SMS/RJ } \\
\hline$<10$ anos $(\mathrm{n}=45)$ & 18 & 40,0 & & 28 & 62,2 & & & 1431,8 & \\
\hline 10 anos ou $+(n=56)$ & 37 & 64,9 & 0,012 & 36 & 64,3 & 0,831 & & 3665,5 & 0,001 \\
\hline \multicolumn{10}{|l|}{ Tipo de Unidade } \\
\hline Hospital $(\mathrm{n}=30)$ & 20 & 66,7 & & 16 & 53,3 & & & 1033,3 & \\
\hline $\operatorname{UBS}^{\mathrm{f}}(\mathrm{n}=55)$ & 26 & 47,3 & & 38 & 69,1 & & & 3361,1 & \\
\hline $\mathrm{USF} / \mathrm{CP} \mathrm{g}(\mathrm{n}=16)$ & 8 & 50,0 & 0,220 & 12 & 75,0 & 0,233 & & 430,8 & 0,020 \\
\hline \multicolumn{10}{|l|}{ Treinamentos SC } \\
\hline $\operatorname{Sim}(\mathrm{n}=79)$ & 47 & 59,5 & & 53 & 6,2 & & & 4151,9 & \\
\hline Não $(\mathrm{n}=23)$ & 8 & 36,4 & 0,054 & 13 & 56,5 & 0,391 & & $\begin{array}{ll}9 & 40,9\end{array}$ & 0,362 \\
\hline \multicolumn{10}{|l|}{ Acesso manuais SC } \\
\hline $\operatorname{Sim}(\mathrm{n}=80)$ & 42 & 52,5 & & 52 & 65,0 & & & $44 \quad 55,7$ & \\
\hline Não $(\mathrm{n}=22)$ & 13 & 59,1 & 0,583 & 14 & 63,6 & 0,906 & & 627,3 & 0,018 \\
\hline \multicolumn{10}{|l|}{ Leitura completa manuais SC } \\
\hline $\operatorname{Sim}(n=45)$ & 29 & 64,4 & & 34 & 75,6 & & & 2659,1 & \\
\hline Não $(\mathrm{n}=35)$ & 13 & 37,1 & 0,015 & 18 & 51,4 & 0,025 & & 1750,0 & 0,423 \\
\hline \multirow[t]{2}{*}{ Exposição/desfecho } & \multicolumn{5}{|c|}{$\begin{array}{c}\text { Abordagem adequada } \\
\text { parceiro }^{\mathrm{d}}\end{array}$} & & \multicolumn{3}{|c|}{$\begin{array}{c}\text { Dificuldades } \\
\text { abordagem DST e }\end{array}$} \\
\hline & & $\mathbf{n}$ & $\%$ & p valo & & & $\mathrm{n}$ & $\%$ & $\mathrm{p}$ valor \\
\hline \multicolumn{10}{|l|}{ Formação } \\
\hline Médico $(\mathrm{n}=71)$ & & 31 & 43,7 & & & & 27 & 38,0 & \\
\hline Enfermeiro $(\mathrm{n}=31)$ & & 19 & 61,3 & 0,101 & & & 20 & 62,5 & 0,021 \\
\hline \multicolumn{10}{|c|}{ Especialização obstetrícia/saúde da mulher } \\
\hline $\operatorname{Sim}(\mathrm{n}=74)$ & & 33 & 44,6 & & & & 28 & 37,3 & \\
\hline Não $(\mathrm{n}=16)$ & & 12 & 75,0 & 0,027 & & & 13 & 81,2 & 0,001 \\
\hline Tempo de formação & & & & & & & & & \\
\hline$<10$ anos $(\mathrm{n}=23)$ & & 14 & 63,6 & & & & 15 & 65,2 & \\
\hline 10 anos ou $+(n=79)$ & & 37 & 46,2 & 0,149 & & & 32 & 40,0 & 0,032 \\
\hline Tempo de atuação SMS/RJ & & & & & & & & & \\
\hline$<10 \operatorname{anos}(\mathrm{n}=45)$ & & 26 & 57,8 & & & & 27 & 60,0 & \\
\hline 10 anos ou $+(n=56)$ & & 23 & 41,1 & 0,095 & & & 19 & 33,3 & 0,007 \\
\hline Tipo de Unidade & & & & & & & & & \\
\hline Hospital $(\mathrm{n}=30)$ & & 16 & 53,3 & & & & 10 & 33,3 & \\
\hline $\mathrm{UBS}^{\mathrm{f}}(\mathrm{n}=55)$ & & 20 & 36,4 & & & & 27 & 50,0 & \\
\hline $\mathrm{USF} / \mathrm{CP}{ }^{\mathrm{g}}(\mathrm{n}=16)$ & & 13 & 86,7 & 0,002 & & & 10 & 62,5 & 0,136 \\
\hline Treinamentos SC & & & & & & & & & \\
\hline $\operatorname{Sim}(\mathrm{n}=79)$ & & 39 & 48,8 & & & & 36 & 45,0 & \\
\hline Não $(\mathrm{n}=23)$ & & 12 & 54,5 & 0,630 & & & 12 & 52,2 & 0,543 \\
\hline Acesso manuais SC & & & & & & & & & \\
\hline $\operatorname{Sim}(\mathrm{n}=80)$ & & 41 & 51,2 & & & & 39 & 48,1 & \\
\hline Não $(\mathrm{n}=22)$ & & 9 & 40,9 & 0,390 & & & 9 & 40,9 & 0,546 \\
\hline Leitura completa manuais SC & & & & & & & & & \\
\hline $\operatorname{Sim}(n=45)$ & & 25 & 54,3 & & & & 17 & 37,8 & \\
\hline Não $(\mathrm{n}=35)$ & & 16 & 47,1 & 0,519 & & & 21 & 60,0 & 0,048 \\
\hline
\end{tabular}

Teste estatístico qui-quadrado. a SC = sífilis congênita; ${ }^{\mathrm{b}}$ Considerada adequada se solicitação do VDRL for realizada na primeira consulta de pré-natal e no início do terceiro trimestre gestacional; ${ }^{c}$ Considerado tratamento adequado se houve acerto do caso clínico apresentado; ${ }^{\mathrm{d} C o n s i d e r a d a}$ abordagem adequada se profissional realizou busca ativa do parceiro para aconselhamento, diagnóstico e tratamento. Considerada conduta inadequada se profissional enviou pedido do exame ou tratamento por meio da gestante; ${ }^{\mathrm{e}}$

Dificuldade abordagem DST = dificuldades relatadas pelo profissional para falar sobre DST em geral, conversar sobre possível forma de infecção com a sífilis, consequências da doença para o bebê e a mulher, orientação sobre tratamento e uso de preservativo, abordagem do parceiro; ${ }^{\mathrm{f}}$ UBS = Unidade Básica de Saúde; ${ }^{\mathrm{g}}$ USF = Unidade de Saúde da Família e CP = Casa de Parto. 
em decorrência da disponibilidade de laboratórios nesses serviços; menor relato de início tardio do pré-natal em USF/Casa de Parto, por acesso facilitado ao atendimento pré-natal nessas unidades de saúde; menor proporção de problemas na referência do parceiro em hospitais e USF/ Casa de parto, por maior disponibilidade de especialistas nos hospitais e atendimento de toda a população por generalistas nas USF. Por outro lado, barreiras relacionadas aos pacientes (não comparecimento do parceiro, não adesão das usuárias) apresentam distribuição semelhante entre os serviços, revelando uma provável homogeneidade da população atendida.

Verificou-se melhor desempenho dos profissionais com maior tempo de formação e de atuação na SMS/RJ em relação às ações de controle da sífilis, provavelmente em decorrência de maior acesso aos treinamentos realizados na última década. Profissionais médicos e aqueles com especialidade em ginecologia/obstetrícia apresentaram menor relato de dificuldade para abordagem das DST, que incluíam fornecimento de informações sobre DST em geral, sobre a forma de transmissão da doença e possíveis consequências da infecção para a saúde da gestante e do bebê, e orientações sobre tratamento e uso de preservativos. A abordagem mais adequada dos parceiros por profissionais sem especialização em obstetrícia/ saúde da mulher está provavelmente associada ao local de atuação, pois a maior parte desses profissionais desenvolvia suas ações nas USF/Casa de Parto (dado não mostrado em tabela).

Maior acesso a treinamentos, manuais e, principalmente, a leitura completa dos manuais, com maior familiaridade dos profissionais com o conteúdo dos protocolos, também mostraram associação com conhecimento correto da situação epidemiológica da sífilis e realização correta das condutas assistenciais. Entretanto, esses efeitos foram discretos e a persistência de falhas nas rotinas assistenciais em profissionais com acesso a treinamentos e manuais normativos indica a necessidade de revisão das estratégias de capacitação que têm sido adotadas.

De um modo geral, estratégias de educação continuada, que são efetivas para mudar a prática dos profissionais e os resultados em saúde, são as que visam aumentar a competência dos profissionais, facilitar a adoção dos protocolos na prática assistencial ou reforçar a mudança ${ }^{18}$. As evidências disponíveis sugerem que a transmissão passiva de informações, por meio de treinamentos tradicionais e divulgação por correio, sejam as estratégias com efeitos mais fracos; au- ditorias e retorno de informações, especialmente quando realizadas no contexto assistencial, de forma concorrente (e não retrospectiva), direcionadas para profissionais específicos e realizadas por pares, apresentam efeito moderado; e intervenções múltiplas, sistemas que relembram os profissionais o que deve ser feito e detalhamento acadêmico - educação individualizada, geralmente feita no consultório médico, por outro profissional de saúde, e mais frequentemente na área de prescrição de medicamento - as que apresentam efeitos fortes ${ }^{18}$.

Estratégias multifacetadas, direcionadas para barreiras distintas, parecem ser mais efetivas do que estratégias isoladas ${ }^{16,22}$, sendo aquelas que requerem participação ativa dos profissionais e que estão mais próximas dos processos decisórios as que apresentam maior chance de sucesso. Em qualquer estratégia, é importante identificar a necessidade de capacitação, verificada por meio da comparação entre a prática observada e aquela desejada $^{18}$.

Neste estudo, os profissionais indicaram os cursos de curta duração, realizados no próprio serviço, como o melhor formato de capacitação. A incorporação de ações de capacitação em aconselhamento nesses treinamentos é essencial, pois além de ter sido sugerida por uma grande parcela dos profissionais, dificuldades em algum aspecto da abordagem das DST foram referidas por quase $50 \%$ dos entrevistados e o acesso a treinamentos e manuais não se mostrou associado à redução dessas dificuldades.

A baixa implantação de protocolos, por falta de confiança dos profissionais em seguir as recomendações, são mais comuns em diretrizes envolvendo aconselhamento e ações educativas ${ }^{19,20}$. Recomendações que envolvem ações de aconselhamento em temas sensíveis, como DST ${ }^{21}$, sexualidade, uso de preservativos, podem ser percebidas como constrangedoras, tanto pelos profissionais como pelos usuários, constituindo-se numa barreira para sua adoção.

O retorno de informações sobre casos de SC ocorridos na cidade e no próprio serviço também deve ser utilizado nas capacitações, embora tenha sido indicada como uma estratégia relevante por apenas $20 \%$ dos profissionais. A falta de confiança nos resultados do protocolo clínico é uma das barreiras relacionadas à atitude dos profissionais $^{19}$, por falta de credibilidade de que a adoção do protocolo resultará em melhores resultados em saúde. O protocolo para manejo da sífilis na gestação atualmente recomendado ${ }^{8}$ visa à eliminação da congênita e, para isso, prevê 
o tratamento de todas as gestantes com exame reagente, mesmo que não confirmado por teste treponêmico, bem como o tratamento de todos os parceiros. Essa conduta pode parecer exagerada para cerca de $30 \%$ dos profissionais que não estão cientes da gravidade da SC na cidade e que provavelmente desconhecem as consequências do manejo inadequado de um caso de sífilis na gestação, sendo uma possível barreira para a adoção correta do tratamento em gestantes com exames reagentes com titulação baixa. A percepção de que as DST não apresentam situação epidemiológica relevante, acoplada à ausência de quadro clínico sugestivo, já foram evidenciadas em outros estudos como razões para a não solicitação rotineira dos exames de triagem ${ }^{23}$.

A baixa adesão das gestantes apontada pelos profissionais, o baixo comparecimento dos parceiros aos serviços e a sugestão de desenvolvimento de trabalhos educativos com a população geral indicam a necessidade de estratégias voltadas também aos usuários, facilitando o acesso a informações em relação às DST e a insumos de prevenção. Estudos que evidenciaram elevadas barreiras relacionadas aos usuários consideram que possa ser útil promover o envolvimento dos pacientes na formulação dos protocolos, bem como no processo decisório ${ }^{20}$, visando aumentar a aplicabilidade e a implantação das recomendações.

Redução de barreiras de acesso aos serviços de pré-natal, à testagem sorológica e ao diagnóstico e tratamento dos parceiros são também fundamentais para o melhor manejo e controle da sífilis na gestação, sendo necessárias estratégias diferentes para os diversos tipos de serviço de saúde.

\section{Conclusão}

O reconhecimento das barreiras específicas na implantação dos protocolos assistenciais tem sido apontado como uma etapa importante na identificação das estratégias a serem adotadas ${ }^{19,20}$. Os resultados deste estudo, realizado em unidades públicas de saúde de uma grande capital brasileira, revelam diversas barreiras relacionadas tanto aos profissionais de saúde quanto aos usuários e ao contexto organizacional, que apresentam distribuição diferente conforme o tipo de serviço de saúde, exigindo abordagens diferenciadas. Destacam-se as barreiras de acesso das gestantes e parceiros ao início precoce da assistência prénatal, à testagem sorológica e ao tratamento adequado da sífilis; e as barreiras relacionadas aos profissionais de saúde, com baixo conhecimento da situação da sífilis congênita na cidade, baixa familiaridade com os protocolos assistenciais e dificuldades na abordagem das DST, principalmente de profissionais sem especialização em obstetrícia/saúde da mulher. A utilização de estratégias multifacetadas ${ }^{16}$, com envolvimento dos usuários dos protocolos, tanto profissionais quanto pacientes, em sua formulação, e a disseminação direcionada dos conteúdos são aspectos importantes a serem considerados na reversão desse quadro.

\section{Colaboradores}

RMSM Domingues e MC Leal participaram da concepção do estudo; delineamento, análise e interpretação dos dados, redação do artigo e aprovação da versão final. V Saraceni participou da concepção do estudo e seu delineamento, da revisão crítica e aprovação da versão final. LM Lauria participou da revisão crítica do artigo e da aprovação da versão a ser publicada. 


\section{Referências}

1. Brasil. Ministério da Saúde (MS). Boletim Epidemiológico Aids-DST 2010; 7(1):45-47 [acessado 2012 jan 19]. Disponível em: http://www.aids.gov.br/publicacao/boletim-epidemiologico-2010.

2. Valderrama J, Zacarias F, Mazin R. Sífilis materna y sífilis congênita em America Latina: um problema grave de solución sencilla. Rev Panam Salud Publica 2004; 16(3):211-217.

3. Ramos Jr AN, Matida LH, Saraceni V, Veras MASM, Pontes RJS. Control of mother-to-child transmission of infectious diseases in Brazil: progress in HIV/Aids and failure in congenital syphilis. Cad Saude Publica 2007; 23(Supl. 3):S370-S378

4. Rio de Janeiro. Secretaria Municipal de Saúde. Sífilis congênita no município do Rio de Janeiro [acessado 2010 jun 20]. Disponível em: http://www. saude.rio.rj.gov.br.

5. Brasil. Ministério da Saúde (MS). Diretrizes para controle da sífilis congênita: manual de bolso. Brasília: Programa Nacional de DST/Aids, MS; 2006.

6. Saraceni V, Guimarães MHSF, Theme Filha MM, Leal MC. Mortalidade perinatal por sífilis congênita: indicador da qualidade da atenção à mulher e à criança. Cad Saude Publica 2005; 21(4):1244-1250.

7. Carroli G, Rooney C, Villar J. How effective is antenatal care in preventing maternal mortality and serious morbidity? An overview of the evidence. Paediatr Perinat Epidemiol 2001; 15(Supl. 1):1-42.

8. Brasil. Ministério da Saúde (MS). Protocolo para a prevenção da transmissão vertical de HIV e sífilis manual de bolso. Brasília: Programa Nacional de Controle de Doenças Sexualmente Transmissíveis e AIDS, MS; 2007.

9. Trepka MJ, Bloom SA, Zhang G, Kim S, Nobles RE. Inadequate syphilis screening among women with prenatal care in a community with a high syphilis incidence. Sex Transm Dis 2006; 33(11):670-674.

10. Rodrigues CS, Guimarães MDC, César CC. Missed opportunities for congenital syphilis and HIV perinatal transmission prevention. Rev Saude Publica 2008; 42(5):851-858.

11. Saraceni V, Domingues RMSM, Vellozo V, Lauria LM, Dias MAB, Ratto KMN, Durovni B. Vigilância da sífilis na gravidez. Epidemiologia e Serviços de Saúde 2007; 16(2):103-111.

12. Domingues RMSM. Avaliação da implantação da assistência pré-natal na rede SUS do Município do Rio de Janeiro com ênfase nas ações de controle da sífilis e do HIV [tese]. Rio de Janeiro: Escola Nacional de Saúde Pública Sérgio Arouca; 2011.

13. Saraceni V, Leal MC. Avaliação da efetividade das campanhas para eliminação da sífilis congênita na redução da morbi-mortalidade perinatal. Município do Rio de Janeiro, 1999-2000. Cad Saude Publica 2003;19(5):1341-1349.

14. Blencowe H, Cousens S, Kamb M, Berman S, Lawn JE. Lives saved tool supplement detection and treatment of syphilis in pregnancy to reduce syphilis related stillbirths and neonatam mortality. BMC Public Health 2011; 11(Supl. 3):S9.
15. Barros FC, Bhutta ZA, Batra M, Hansen TN, Victora CG, Rubens CE, and the GAPPS review group. Global report on preterm and stillbirth (3 of 7): evidence for effectiveness of interventions. $B M C$ Pregnancy Childbirth 2010; 10(Supl. 1):S3.

16. Francke AL, Smit MC, de Veer AJE, Mistiaen P. Factors influencing the implementation of clinical guidelines for health care professionals: A systematic metareview. BMC Med Inform Decis Mak 2008; 8:38.

17. Carlsen B, Glenton C, Pope C. Thou shalt versus thou shalt not: a meta-synthesis of GPs' attitudes to clinical practice guidelines. Br J Gen Pract 2007; 57(545):971-978.

18. Davis DA, Taylor-Vaisey A. Translating guidelines into practice. A systematic review of theoretic concepts, practical experience and research evidence in the adoption of clinical practice guidelines. CMAJ 1997; 157(4):408-416.

19. Cabana MD, Rand CS, Powe NR, Wu AW, Wilson MH, Abboud PA, Rubin HR. Why don't physicians follow clinical practice guidelines? A framework for improvement. JAMA 1999; 282(15):1458-1465.

20. Lugtenberg M, Burgers JS, Besters CF, Han D, Westert GP. Perceived barriers to guideline adherence: a survey among general practitioners. BMC Fam Pract. 2011; 12:98.

21. Lugtenberg M, Zegers-van Schaick JM, Westert GP, Burgers JS. Why don't physicians adhere to guideline recommendations in practice? An analysis of barriers among Dutch general practitioners. Implement Sci. 2009; 4:54.

22. Grimshaw JM, Shirran L, Thomas R, Mowatt G, Fraser C, Bero L, Grilli R, Harvey E, Oxman A, O'Brien MA. Changing provider behavior: an overview of systematic reviews of interventions. Med Care 2001; 39(8 Supl. 2):II2-45.

23. Todd CS, Ahmadzai M, Smith JM, Siddiqui H, Ghazanfar SA, Strathdee SA. Attitudes and practices of obstetric care providers in Kabul, Afghanistan regarding antenatal testing for sexually transmitted infection. J Obstet Gynecol Neonatal Nurs 2008; 37(5):607-615.

Artigo apresentado em 04/04/2012

Aprovado em 18/08/2012

Versão final apresentada em 04/09/2012 